
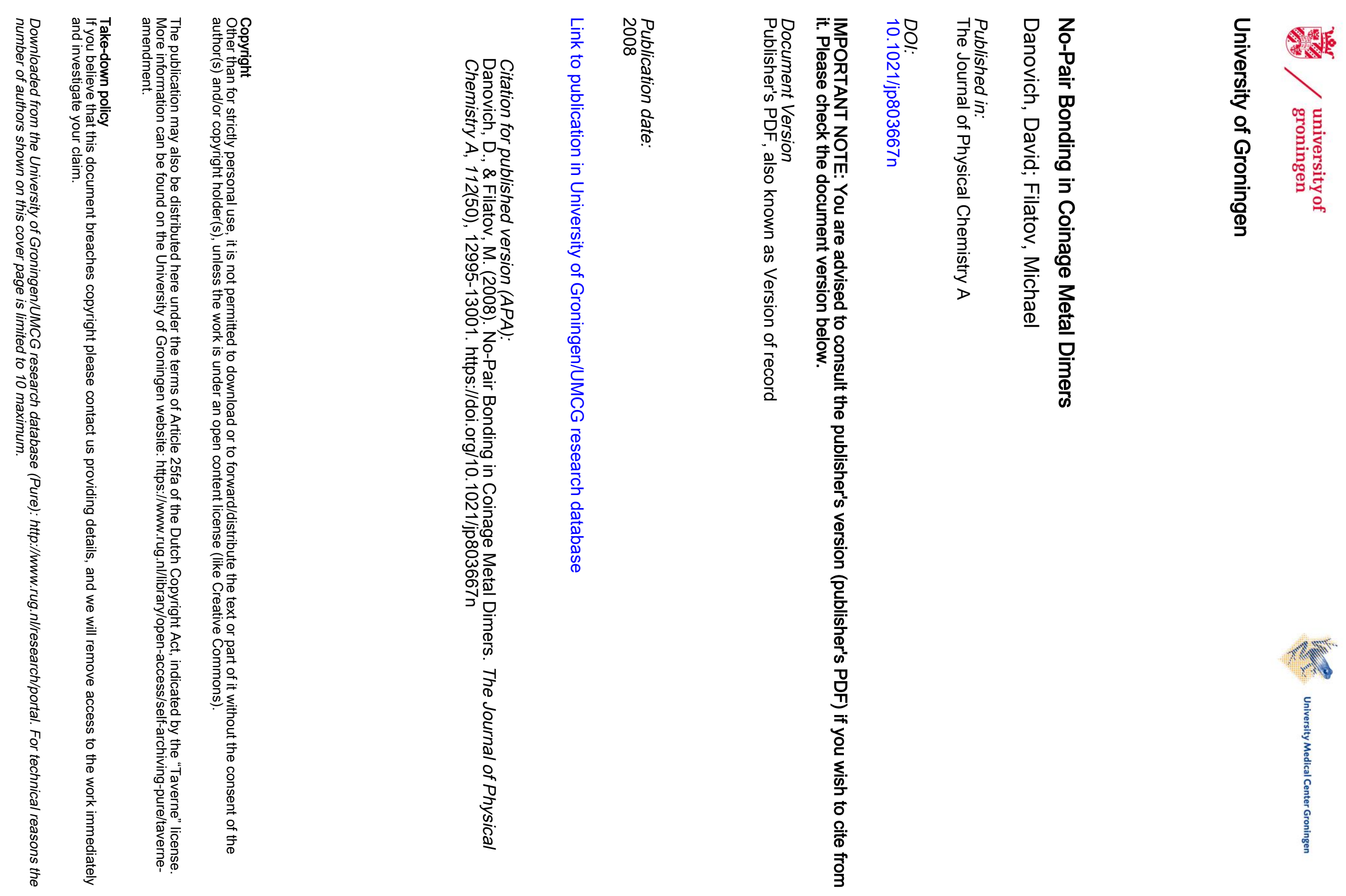


\title{
No-Pair Bonding in Coinage Metal Dimers ${ }^{\dagger}$
}

\author{
David Danovich* \\ The Lise-Meitner Minerva Center for Computational Quantum Chemistry, The Hebrew University, \\ Jerusalem 91904, Israel
}

\author{
Michael Filatov \\ Theoretical Chemistry, Zernike Institute for Advanced Materials, University of Groningen, Nijenborgh 4, \\ 9747 AG Groningen, The Netherlands
}

Received: April 27, 2008; Revised Manuscript Received: June 12, 2008

\begin{abstract}
High-level ab initio calculations at the coupled cluster with single and double substitutions and perturbative treatment of triple substitutions, $\operatorname{CCSD}(\mathrm{T})$, level of theory have been carried out for the dimers of coinage metal atoms $\mathrm{Cu}, \mathrm{Ag}$, and $\mathrm{Au}$ in the ground ${ }^{1} \Sigma_{\mathrm{g}}^{+}$state and in the excited ${ }^{3} \Sigma_{\mathrm{u}}^{+}$state. All of the calculations have been carried out with the inclusion of scalar-relativistic effects via the normalized elimination of the small component (NESC) method. For the dimers in the triplet state, nonzero bond dissociation energies are obtained which vary from $1.3 \mathrm{kcal} / \mathrm{mol}$ for ${ }^{3} \mathrm{Cu}_{2}$ to $4.6 \mathrm{kcal} / \mathrm{mol}$ for ${ }^{3} \mathrm{Au}_{2}$. Taking into account that, in bulky high-spin copper clusters, the bond dissociation energy per atom increases steeply to the value of ca. $19 \mathrm{kcal} / \mathrm{mol}$, the results obtained in the present paper suggest that the bond dissociation energy per atom in high-spin gold clusters may reach extremely high values exceeding $20 \mathrm{kcal} / \mathrm{mol}$ thus becoming comparable to the usual bonding due to the spin-pairing mechanism.
\end{abstract}

\section{Introduction}

No-pair or ferromagnetic bonding ${ }^{1,2,4-8}$ in high-spin alkali metal clusters is a type of bonding which defies a paradigm that strong chemical bond may result only from singlet pairing of the electron spins as was introduced in chemistry by Lewis. ${ }^{9}$ Despite that, in these clusters, spins of all valence electrons are aligned in parallel, according to density functional calculations they are bound by energies reaching as much as $12 \mathrm{kcal} / \mathrm{mol}$ per atom. ${ }^{4-8}$ Another peculiar feature of no-pair bonding is in the nonadditivity of binding energy in high-spin clusters, which varies between less than $1 \mathrm{kcal} / \mathrm{mol}$ per atom in diatomic molecules and reaches relatively high values in large clusters. ${ }^{4-8,16,10,11}$ A thorough analysis carried out by Shaik et al. ${ }^{5-7}$ showed that no-pair bonding can be best described as resulting from ionic fluctuations of the triplet electronic pairs that spread over all the close neighbors in the cluster and lead to a rapid increase in the binding energy in bulky three-dimensional structures.

It is noteworthy that the study of no-pair bonding is not driven by the chemical curiosity alone. There is an interest in studying this type of bonding stimulated by the properties of BoseEinstein condensates in dilute gases of alkali metal atoms. ${ }^{12-14}$ There is also a growing interest in investigation of magnetic clusters which may find a large number of potential applications in medicine and nanoscience. ${ }^{15}$ In the quest for new systems exhibiting no-pair bonding, one moves naturally to late transition metals such as coinage metals which possess a closed d-shell and a singly occupied s-shell thus bearing similarity with the electronic structure of alkali metals. In the first exploration of this terrain, ${ }^{16}$ it was found that copper can make high-spin clusters devoid of electron pairs which are bound by as much

\footnotetext{
Part of the "Sason S. Shaik Festschrift".

* To whom correspondence should be addressed. E-mail: david.danovich@ huji.ac.il.

‡E-mail: m.filatov@ @ug.nl.
}

as $19 \mathrm{kcal} / \mathrm{mol}$ per atom, which is ca. $50 \%$ greater than in the corresponding lithium clusters. These no-pair clusters are not merely theoretical curiosities for the purpose of demonstrating unusual bonding features; some of these clusters have actually been made and probed by experimental techniques. ${ }^{17-19}$

In the present work, we attempt to extend the domain of search for no-pair bonding in coinage metal clusters by investigating the high-spin copper, silver, and gold clusters with high-level ab initio wave function methods. Because, in heavy coinage metal atoms, relativistic effects are very important for the correct description of their electronic structure, a relativistic all-electron formalism is employed in connection with highly correlated wave function method in order to calculate the potential energy curves of coinage metal dimers in the ${ }^{3} \Sigma_{\mathrm{u}}^{+}$state. The results of these calculations will be compared with the available experimental estimates for the spectroscopic parameters of the high-spin dimeric species ${ }^{20}$ and with the properties of coinage metal dimers in the ground ${ }^{1} \Sigma_{\mathrm{g}}^{+}$state. It is the primary purpose of the present work to provide high quality data which can be used as benchmarks in the further study of bigger highspin coinage metal clusters.

\section{Details of Calculations}

Calculations of the potential energy curves of the $\mathrm{Cu}, \mathrm{Ag}$, and $\mathrm{Au}$ dimers in the singlet ${ }^{1} \Sigma_{\mathrm{g}}^{+}$ground-state and in the lowest triplet $a-{ }^{3} \Sigma_{\mathrm{u}}^{+}$excited-state have been carried out with the use of the coupled cluster with single and double substitutions and with perturbational treatment of triple substitutions, $\operatorname{CCSD}(\mathrm{T})$, method. The zero order wave functions were obtained in the spin-restricted Hartree-Fock calculations for the closed-shell species and in the spin-unrestricted Hartree-Fock calculations for the open-shell species. The bond dissociation energies were calculated with respect to the ground states of neutral atoms. The counterpoise correction of Boys and Bernardi ${ }^{21}$ was employed to eliminate the basis set superposition error. 
TABLE 1: Spectroscopic Parameters of Copper Dimers in Singlet ${ }^{1} \Sigma_{\mathrm{g}}^{+}$and in Triplet ${ }^{3} \boldsymbol{\Sigma}_{\mathrm{u}}^{+}$States Calculated with the CCSD(T) Method Using Relativistically Corrected NESC Hamiltonian ${ }^{a}$

\begin{tabular}{|c|c|c|c|c|c|c|}
\hline molecule & state & $D_{\mathrm{e}}, \mathrm{kcal} / \mathrm{mol}$ & $R_{\mathrm{e}}, \AA$ & $\omega_{\mathrm{e}}, \mathrm{cm}^{-1}$ & BSSE & basis set \\
\hline \multirow[t]{4}{*}{$\mathrm{Cu}_{2}$} & ${ }^{1} \sum_{\mathrm{g}}^{+}$ & 44.9 & 2.229 & 265.0 & no & aug-cc-pVTZ ${ }^{b, c}$ \\
\hline & & 44.8 & 2.231 & 261.9 & no & aug-cc-pVQZ \\
\hline & & 44.9 & 2.230 & 262.9 & no & aug-cc-pV5Z \\
\hline & & 44.9 & 2.231 & 261.7 & no & extrap. CBS $^{d}$ \\
\hline \multirow[t]{4}{*}{$\mathrm{Cu}_{2}$} & ${ }^{1} \Sigma_{\mathrm{g}}^{+}$ & 44.1 & 2.235 & 262.9 & yes & aug-cc-pVTZ \\
\hline & & 44.4 & 2.236 & 260.6 & yes & aug-cc-pVQZ \\
\hline & & 44.6 & 2.234 & 262.2 & yes & aug-cc-pV5Z \\
\hline & & 44.7 & 2.235 & 261.3 & yes & extrap. CBS \\
\hline exptl. & & $46.4^{e}$ & $2.2197^{e}$ & $266^{e}$ & & \\
\hline \multirow[t]{4}{*}{$\mathrm{Cu}_{2}$} & ${ }^{3} \sum_{\mathrm{u}}^{+}$ & $1.27(0.66)$ & $2.711(2.965)$ & $65.1(32.5)$ & no & aug-cc-pVTZ \\
\hline & & $1.29(0.70)$ & $2.725(2.960)$ & $65.3(34.2)$ & no & aug-cc-pVQZ \\
\hline & & $1.28(0.70)$ & $2.724(2.965)$ & 63.9 & no & aug-cc-pV5Z \\
\hline & & 1.27 & 2.731 & 63.2 & no & extrap. CBS \\
\hline \multirow[t]{4}{*}{$\mathrm{Cu}_{2}$} & ${ }^{3} \Sigma_{\mathrm{u}}^{+}$ & $0.86(0.39)$ & $2.761(3.205)$ & 54.8 & yes & aug-cc-pVTZ \\
\hline & & $1.13(0.59)$ & $2.755(3.020)$ & 59.4 & yes & aug-cc-pVQZ \\
\hline & & $1.15(0.61)$ & $2.752(3.035)$ & 58.3 & yes & aug-cc-pV5Z \\
\hline & & 1.22 & 2.756 & 58.6 & yes & extrap. CBS \\
\hline exptl. & & $2.86-4.28^{f}$ & $2.48 \pm 0.03^{f}$ & $125 \pm 25^{f}$ & & \\
\hline
\end{tabular}

${ }^{a}$ The results of non-relativistic $\operatorname{CCSD}(\mathrm{T})$ calculations are given in parentheses. ${ }^{b}$ Augmented correlation consistent basis sets from ref 22. ${ }^{c}$ All basis sets are used in uncontracted form. ${ }^{d}$ The SCF energy is extrapolated using exponential formula and the correlation energy is extrapolated using cubic formula. ${ }^{e}$ Taken from ref $34 .{ }^{f}$ From ref 20.

In the calculations on copper dimers, the correlation consistent augmented basis sets of triple- $\zeta$ (aug-cc-pVTZ-DK), quadruple- $\zeta$ (aug-cc-pVQZ-DK), and pentuple- $\zeta$ (aug-cc-pV5Z-DK) quality were employed. ${ }^{22}$ These basis sets were totally uncontracted in the calculations. Because these basis sets form a systematic sequence, it was possible to extrapolate the total energy to the complete basis set limit. ${ }^{23,24}$ In the extrapolation procedure, the Hartree-Fock total energy was extrapolated with the use of a three-parameter exponential model $a+b e^{-c X},{ }^{23}$ and the $\operatorname{CCSD}(\mathrm{T})$ correlation energy was extrapolated with the use of a cubic model $A+B X^{-3},{ }^{24}$ where $X$ is the cardinal number of the basis set (i.e., three for aug-cc-pVTZ, four for aug-cc-pVQZ, and five for aug-cc-pV5Z).

Silver and gold dimers were calculated with the use of the double- $\zeta$ (DZ) and triple- $\zeta$ (TZ) quality basis sets of Dyall. ${ }^{25}$ These basis sets were used in uncontracted form and augmented with valence polarizing and valence correlating functions as suggested in ref 25 . Thus, the following basis sets resulted: DZ(Ag) (21s14p10d2f) basis set, TZ(Ag) (28s20p13d3f) basis set, and $\mathrm{DZ}(\mathrm{Au})(22 \mathrm{~s} 19 \mathrm{p} 12 \mathrm{~d} 9 \mathrm{f})$ basis set. The basis sets employed in the present work have been specifically designed for all-electron relativistic calculations. ${ }^{25}$

The calculations have been carried out at the nonrelativistic $\operatorname{CCSD}(\mathrm{T})$ level and with the inclusion of scalar-relativistic effects via the normalized elimination of the small component (NESC) method. ${ }^{26}$ The iterative solution of the NESC equations as described in ref 27 was implemented in the COLOGNE08 package. ${ }^{28}$ Because the relativistic integral code does not support basis functions with the angular momentum higher than 3 , the $\mathrm{G}, \mathrm{H}$, and I primitive functions were dropped from the aug-ccpVTZ-DK, aug-cc-pVQZ-DK, and aug-cc-pV5Z-DK basis sets. In order to evaluate the effect of higher angular momentum functions we have also done quasi-relativistic calculations using Douglas-Kroll-Hess (DKH) quasi-relativistic Hamiltonian ${ }^{29}$ implemented in MOLPRO2006.1 package. ${ }^{30}$ In these calculations, the uncontracted aug-cc-pVTZ-DK basis set was used. Calculations at the CCSDT level, at which the amplitudes of the triple excitations are obtained iteratively, have been carried out for copper dimers with the use of the relativistic effective core potentials (RECPs) in order to estimate the effect of higherorder excitations not fully covered at the $\operatorname{CCSD}(\mathrm{T})$ level.
TABLE 2: Equilibrium Bond Lengths $R_{\mathrm{e}}$ (in $\AA$ ) and Bond Dissociation Energies $D_{\mathrm{e}}$ (in $\mathrm{kcal} / \mathrm{mol}$ ) for the ${ }^{3} \boldsymbol{\Sigma}_{\mathrm{u}}^{+}$State of the Copper Dimer Calculated with the aug-cc-pVTZ-DK Basis Set with and without Primitive G Functions ${ }^{a}$

\begin{tabular}{lccccc}
\hline & \multicolumn{2}{c}{ nonrelativistic } & & \multicolumn{2}{c}{ quasi-relativistic DKH } \\
\cline { 2 - 3 } \cline { 5 - 6 } & $R_{\mathrm{e}}$ & $D_{\mathrm{e}}$ & & $R_{\mathrm{e}}$ & $D_{\mathrm{e}}$ \\
\hline without G functions & 2.965 & 0.664 & & 2.716 & 1.219 \\
with G functions & 2.915 & 0.727 & & 2.685 & 1.333
\end{tabular}

${ }^{a}$ The calculations are carried out with the use of the non-relativistic $\operatorname{CCSD}(\mathrm{T})$ method and with the use of the quasi-relativistic DKH/ $\operatorname{CCSD}(\mathrm{T})$ method.

Because the CCSDT method is much more computationally demanding than $\operatorname{CCSD}(\mathrm{T})$, the all-electron calculations at this level for other dimers are not currently feasible. All of the nonrelativistic and RECP calculations have been carried out with the use of the Gaussian 03 package ${ }^{31}$ except for the CCSDT ones which have been done with the use of the NWCHEM5.0 package. ${ }^{32}$

\section{Results and Discussion}

The bond dissociation energies (BDEs), bond lengths, and harmonic vibrational frequencies of copper dimer in the singlet and the lowest triplet states calculated with different basis sets are collected in Table 1. In this table, the spectroscopic parameters obtained with and without the account of basis set superposition error (BSSE) are reported. Comparison of the BSSE corrected and uncorrected results shows clearly that there is a convergence in the calculated properties with basis sets of increasing size. The difference between the values obtained with BSSE and without BSSE reduces to less than $0.3 \mathrm{kcal} / \mathrm{mol}$ for the energies, $0.004 \AA$ for the bond lengths, and $0.7 \mathrm{~cm}^{-1}$ for the vibrational frequencies in the calculations employing the aug-cc-pV5Z basis set. The values extrapolated to the complete basis set (CBS) show similarly good convergence.

The so-obtained spectroscopic parameters of the singlet copper dimer are in fairly good agreement with the experimental data. The remaining discrepancy in BDE of $1.6 \mathrm{kcal} / \mathrm{mol}$ can be further reduced in the coupled-cluster calculations with the full iterative account of triple excitations, which is achieved in 


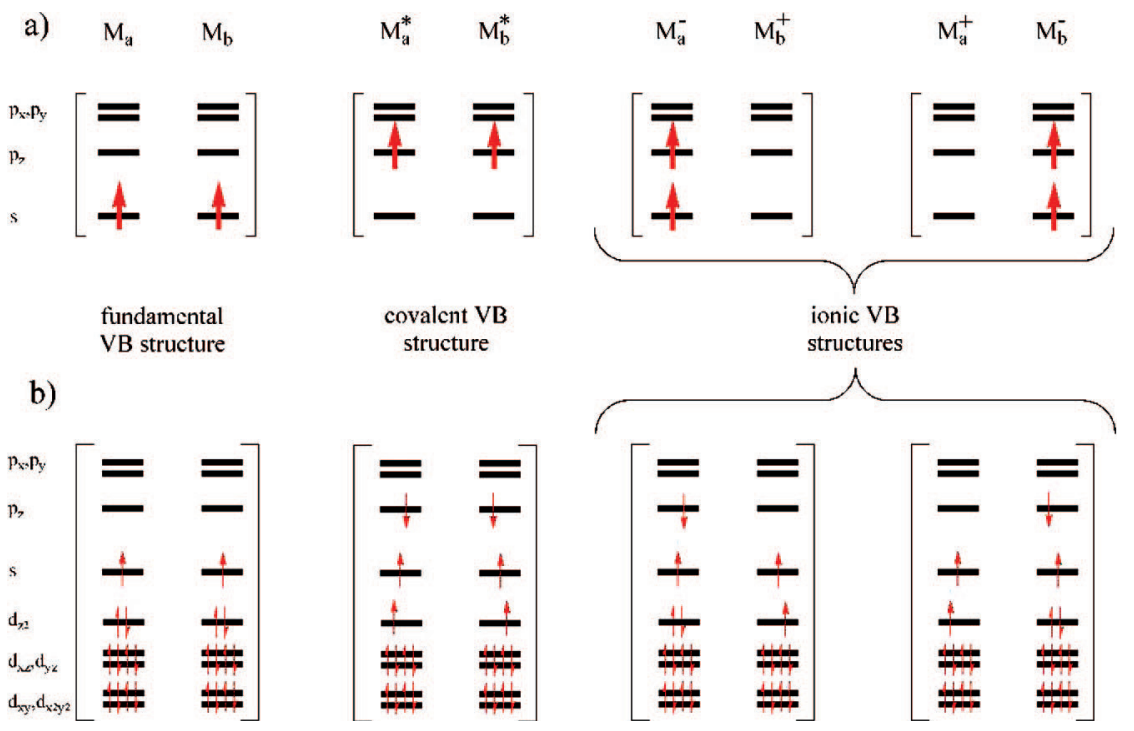

Figure 1. Scaematic representation of valence bond (VB) structures contributing to the no-pair bonding in high-spin alkali metal dimers (panel a) and in high-spin coinage metal dimers (panel b). The fundamental VB structure is repulsive, the covalent VB structures lead to dispersion-like attraction, and the charge transfer VB structures result in additional electrostatic attraction. ${ }^{8}$ Note that, in panel $\mathrm{b}$, only the structures which are specific to coinage metal dimers are shown, that is the full set of no-pair VB structures in coinage metal dimers includes those shown in panels a and $b$.

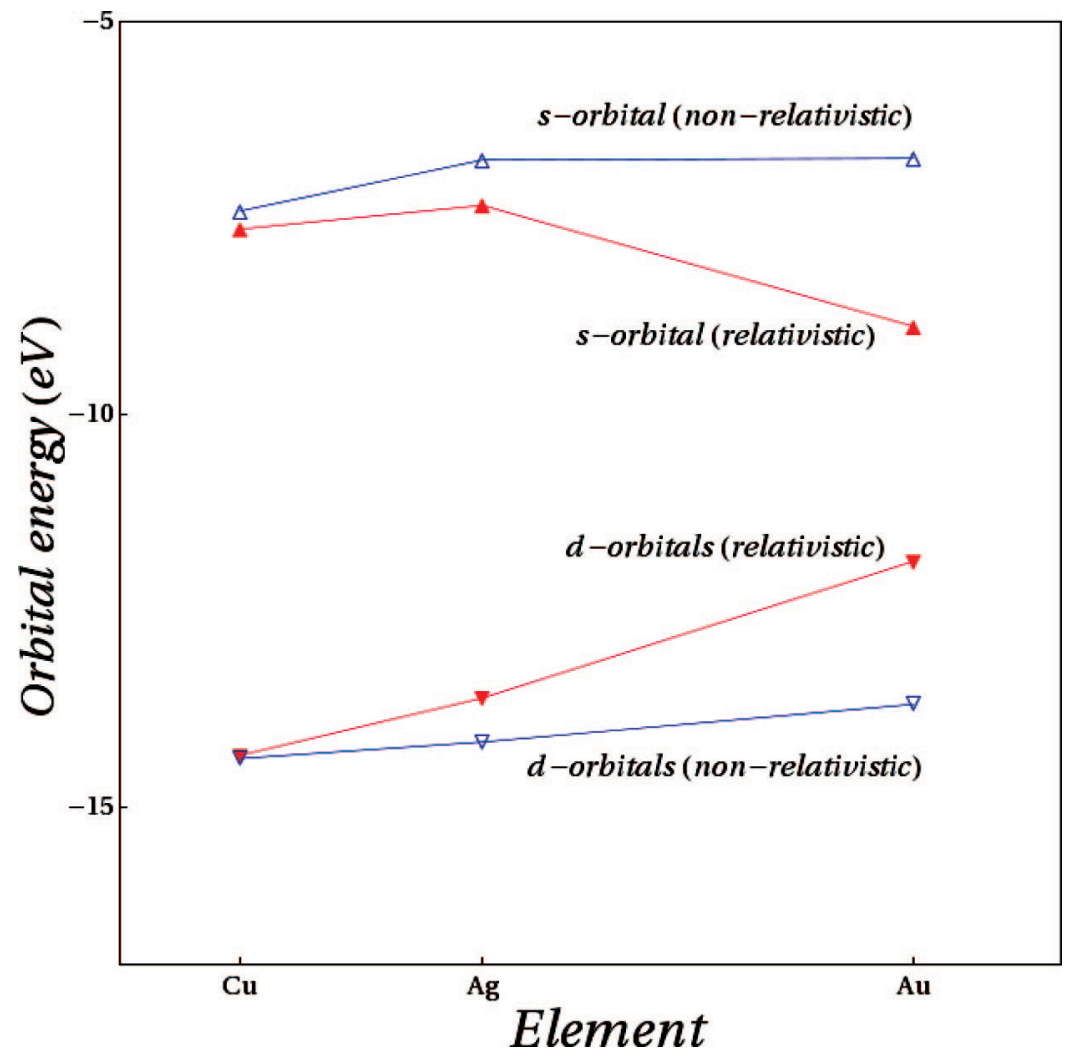

Figure 2. Energies $(\mathrm{eV})$ of valence $\mathrm{s}$ and d orbitals of coinage metal atoms as obtained in the OVGF calculations with and without the use of the relativistic Hamiltonian (see text for detail).

the CCSDT method. A clear indication that this should be the case is provided by comparison of the results of $\operatorname{CCSD}(\mathrm{T})$ and CCSDT calculations carried out with the use of the Stuttgart relativistic effective core potential with extended valence basis set in $(8 \mathrm{~s} 7 \mathrm{p} 6 \mathrm{~d}) /[6 \mathrm{~s} 5 \mathrm{p} 3 \mathrm{~d}]$ contraction. ${ }^{33}$ In these calculations, the ${ }^{1} \mathrm{Cu}_{2} \mathrm{BDE}$ is increased by $1.2 \mathrm{kcal} / \mathrm{mol}$ with the full iterative account of triple excitations. However, the all-electron calculations with CCSDT do not seem currently feasible. In any case, the present $\operatorname{CCSD}(\mathrm{T})$ results for ${ }^{1} \mathrm{Cu}_{2}$ are in better agreement with the experiment than the previously obtained values, ${ }^{16}$ which differed from the experimental values by almost $5 \mathrm{kcal} / \mathrm{mol}$ for BDE and by $0.06 \AA$ for bond length. Note that, in the present work, we employ an all-electron relativistic formalism, whereas in the previous study a nonrelativistic approach was used. ${ }^{16}$ Hence, the good agreement with the experiment obtained in the present work can also indicate the importance of including relativistic effects for accurate description of molecular properties even in the case of an element as light as copper $(Z=29)$. Clearly, such an account is mandatory for heavier homologue such as silver and gold dimers. 
TABLE 3: Spectroscopic Parameters of Silver and Gold Dimers in Singlet ${ }^{1} \boldsymbol{\Sigma}_{\mathrm{g}}^{+}$and in Triplet ${ }^{3} \boldsymbol{\Sigma}_{\mathrm{u}}^{+}$States Calculated with the CCSD(T) Method Using Relativistically Corrected NESC Hamiltonian

\begin{tabular}{lcccccc}
\hline molecule & state & $D_{\mathrm{e}}, \mathrm{kcal} / \mathrm{mol}$ & $R_{\mathrm{e}}, \AA$ & $\omega_{\mathrm{e}}, \mathrm{cm}^{-1}$ & $\mathrm{BSSE}$ & basis set \\
\hline $\mathrm{Ag}_{2}$ & ${ }^{1} \Sigma_{\mathrm{g}}^{+}$ & 36.8 & 2.578 & 183.7 & no & $\mathrm{DZ}^{a, b}$ \\
& & 35.8 & 2.561 & 187.6 & no & $\mathrm{TZ}^{c}$ \\
$\mathrm{Ag}_{2}$ & ${ }^{1} \Sigma_{\mathrm{g}}^{+}$ & 34.5 & 2.587 & 180.9 & yes & $\mathrm{DZ}$ \\
& & 35.3 & 2.574 & 183.8 & yes & $\mathrm{TZ}$ \\
exptl. & & $38.5^{d}$ & $2.530^{e}$ & $192^{d}$ & & \\
$\mathrm{Ag}_{2}$ & ${ }^{3} \Sigma_{\mathrm{u}}^{+}$ & 1.51 & 3.218 & 39.3 & no & $\mathrm{DZ}$ \\
& & 0.74 & 3.566 & 15.3 & no & $\mathrm{TZ}$ \\
$\mathrm{Ag}_{2}$ & ${ }^{3} \Sigma_{\mathrm{u}}^{+}$ & 0.56 & 3.616 & 16.5 & yes & $\mathrm{DZ}$ \\
& & 0.68 & 3.674 & 13.6 & yes & $\mathrm{TZ}$ \\
$\mathrm{Au}_{2}$ & ${ }^{1} \Sigma_{\mathrm{g}}^{+}$ & 51.9 & 2.509 & 184.7 & no & $\mathrm{DZ}$ \\
& & 48.0 & 2.525 & 179.0 & yes & $\mathrm{DZ}$ \\
exptl. & & $53.3^{d}$ & $2.472^{d}$ & $191^{d}$ & & \\
$\mathrm{Au}_{2}$ & ${ }^{3} \sum_{u}^{+}$ & 7.48 & 2.884 & 70.0 & no & $\mathrm{DZ}$ \\
& & 4.56 & 2.942 & 59.2 & yes & $\mathrm{DZ}$
\end{tabular}

${ }^{a}$ Double-zeta quality basis set from ref $25 .{ }^{b}$ All basis sets are used in uncontracted form. ${ }^{c}$ Triple-zeta quality basis set from ref 25. ${ }^{d}$ Taken from ref $36 .{ }^{e}$ From ref 35 .

The importance of the account of relativity for accurate description of molecular properties is even more pronounced in the triplet state of $\mathrm{Cu}_{2}$. For this state, the results of both relativistically corrected all-electron calculations and nonrelativistic all-electron calculations are reported in Table 1. Relativity leads to a 2-fold increase in BDE and in vibrational frequency and in a shortening of the $\mathrm{Cu}-\mathrm{Cu}$ bond length by more than $0.2 \AA$. Similarly to the case of the singlet copper dimer, the spectroscopic constants obtained in the $\operatorname{CCSD}(\mathrm{T})$ calculations show convergence to the same values with extension of the basis sets (see Table 1). The CBS extrapolated values obtained in the series of calculations with the account of BSSE and without the account of BSSE differ by less than $0.05 \mathrm{kcal} / \mathrm{mol}$ for the BDEs.

The discrepancy with the experimentally measured values for the spectroscopic parameters of the triplet $\mathrm{Cu}_{2}$ is more substantial. It should however be noted, that the experimentally measured values reported in Table 1 were obtained from laser spectroscopy measurements on matrix isolated copper dimers. ${ }^{20}$ The major source of uncertainty in these experiments is in the influence of the matrix environment on the observed parameters of spectroscopic transitions. ${ }^{20}$ Although chemically inert matrices (neon or argon) were used, their influence on the properties of the embedded molecular species is non-negligible. ${ }^{37}$ At the present, $\operatorname{CCSD}(\mathrm{T})$, level of theoretical description, it was not possible to include any rare gas atoms in the computed models. However, the high accuracy of the description of spectroscopic parameters of the singlet copper dimer gives us confidence that the theoretical values reported in Table 1 represent reasonably accurate estimates of the parameters of gas phase ${ }^{3} \mathrm{Cu}_{2}$ molecules.

Besides matrix effects, which are relatively difficult to quantify, the theoretical values in Table 1 may be affected by an insufficient level of treatment of the electron correlation as provided by the $\operatorname{CCSD}(\mathrm{T})$ method. Comparison between the results of $\operatorname{CCSD}(\mathrm{T})$ and $\mathrm{CCSDT}$ calculations carried out for ${ }^{3} \mathrm{Cu}_{2}$ with the use of Stuttgart RECP shows that the full iterative account of triple excitations in CCSDT increases the BDE by as much as $0.8 \mathrm{kcal} / \mathrm{mol}$. Thus, be it possible to carry out the all-electron relativistically corrected CCSDT calculations, the theoretical BDE might be somewhat closer to the experimental estimate. The $\mathrm{Cu}-\mathrm{Cu}$ bond length shortening of $0.205 \AA$ was obtained in the RECP-CCSDT calculation as compared to the RECP-CCSD(T) one. Assuming that increments of similar magnitude might be obtained in the all-electron calculations, one can estimate that the use of the CCSDT method might have resulted in the ${ }^{3} \mathrm{Cu}_{2}$ BDE of ca. $2 \mathrm{kcal} / \mathrm{mol}$ and the bond length of $2.55-2.53 \AA$. It is noteworthy that a similar magnitude of the higher-order correlation effects was obtained recently by Monari et al. ${ }^{38}$ on the basis of comparison of the $\operatorname{CCSD}(\mathrm{T})$ and the full CI bond dissociation energies in high-spin tetrahedral $\mathrm{Li}_{4}$ clusters.

As has been pointed out in the preceding section, the relativistic integral code does not currently support basis functions with angular momentum higher than 3 . Therefore, to evaluate the effect of neglecting Gaussian primitive functions with higher angular momentum a series of nonrelativistic and quasi-relativistic calculations for the ${ }^{3} \mathrm{Cu}_{2}$ dimer which employed the fully uncontracted aug-cc-pVTZ-DK basis set were carried out. These calculations were carried out with the use of the $G$ primitive functions as in the original aug-cc-pVTZ-DK basis set and without these functions. The results of the quasirelativistic DKH and nonrelativistic calculations are presented in Table 2. In both cases, the difference in the equilibrium distances does not exceed $0.05 \AA$ and the difference in the BDEs is ca. $0.1 \mathrm{kcal} / \mathrm{mol}$, where a slightly shorter and stronger bond is obtained with the inclusion of the higher angular momentum basis functions. We can therefore conclude that the inclusion of the omitted functions in the NESC calculations should not alter considerably the results reported in Table 1 .

The huge effect of relativity on the spectroscopic parameters of ${ }^{3} \mathrm{Cu}_{2}$ warrants some discussion. As it has been analyzed previously by De Visser et al., ${ }^{16}$ bonding in the triplet copper dimer (likewise to the high-spin alkali metal dimers) is attributed to the so-called no-pair (or ferromagnetic) bonding mechanism. In alkali metals, the no-pair bonding arises due to the following two types of virtual excitations shown schematically in Figure 1a: excitations from the atomic valence s-orbitals to the valence p-orbitals on the same atom, which result in fluctuating local dipole moments at each atomic center, and charge transfer excitations where the $\mathrm{s}$ valence electron on one metal atom is excited to the empty p-orbital on the neighboring atom. ${ }^{4-7,16,8}$ A combination of the two types of excitations leads to a relatively strong bonding, which in metal clusters of large size exceeds the usual Van der Waals interaction energy by a wide margin reaching values of BDE per atom of as much as $19 \mathrm{kcal} /$ mol according to B3P86 calculations with Stuttgart RECP. ${ }^{16}$

In coinage metal atoms, the no-pair bonding originates due to the same mechanism as in alkali metal atoms, that is due to the ionic-covalent fluctuations of the triplet pair. ${ }^{16}$ However, in addition to the $s-p$ excitations, excitations from the valence d-orbitals to the singly occupied s- and the vacant p-orbitals give rise to a number of covalent and ionic valence bond structures shown schematically in Figure $1 \mathrm{~b}$ which also contribute to the no-pair bonding. Thus, the bonding in high-spin coinage metal clusters is expected to be stronger than in the respective alkali metal clusters. Soldán et al. ${ }^{10}$ using the RECP/ $\operatorname{CCSD}(\mathrm{T})$ calculations have obtained the following BDEs for the alkali metal dimers in the triplet state: ${ }^{3} \mathrm{Li}_{2}, 0.955 \mathrm{kcal} / \mathrm{mol}$; ${ }^{3} \mathrm{Na}_{2}, 0.498 \mathrm{kcal} / \mathrm{mol} ;{ }^{3} \mathrm{~K}_{2}, 0.722 \mathrm{kcal} / \mathrm{mol},{ }^{3} \mathrm{Rb}_{2}, 0.633 \mathrm{kcal} /$ $\mathrm{mol}$, and ${ }^{3} \mathrm{Cs}_{2}, 0.706 \mathrm{kcal} / \mathrm{mol}$. In comparison with the potassium ${ }^{3} \mathrm{~K}_{2}$ and rubidium ${ }^{3} \mathrm{Rb}_{2}$ dimers, high-spin copper dimer is bound by almost twice as strong bond as these molecules. This comparison shows that excitations from the valence d-orbitals, which do not contribute to the no-pair bonding in the alkali 

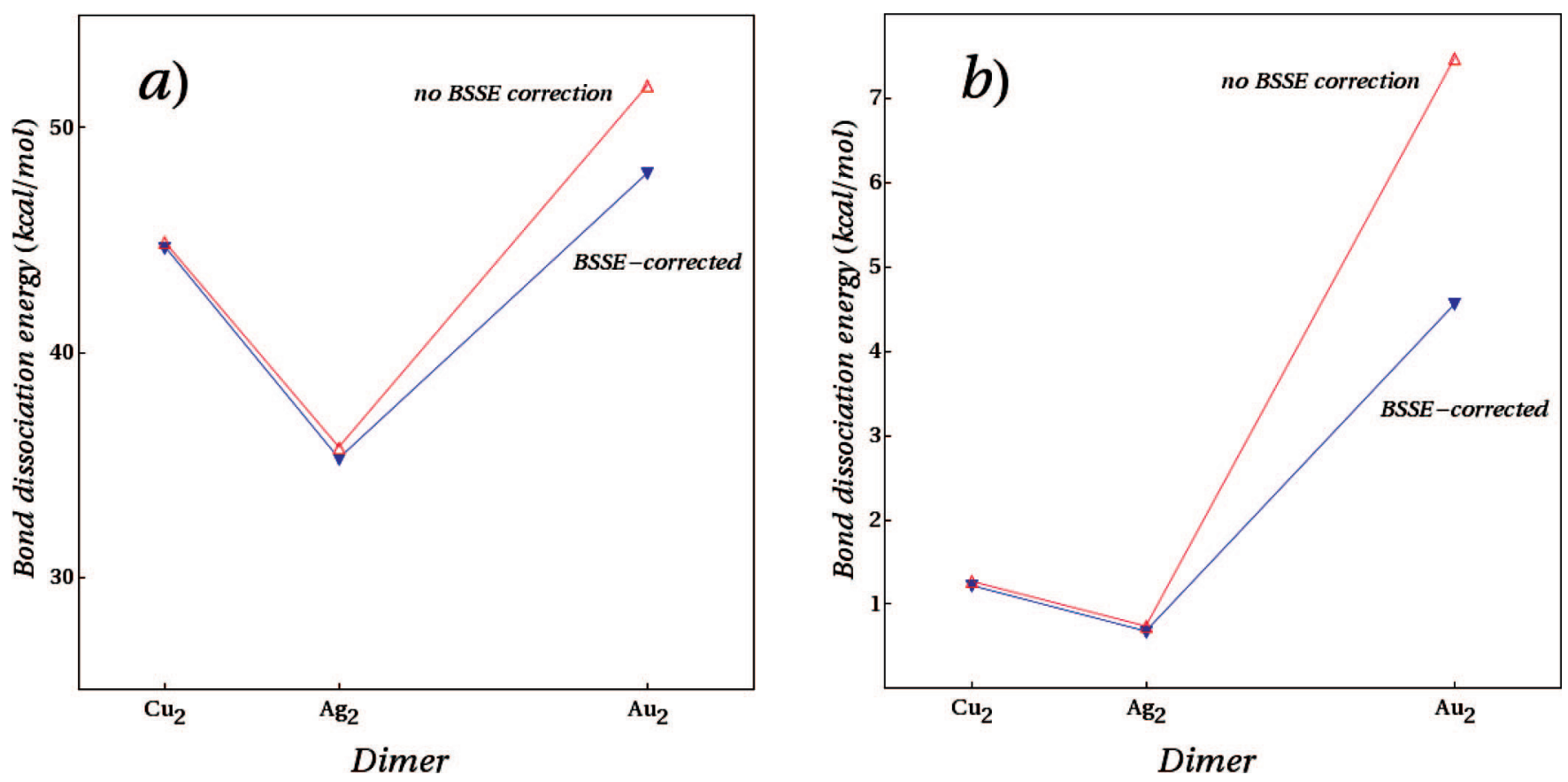

Figure 3. Bond dissociation energies $\left(\mathrm{kcal} / \mathrm{mol}\right.$ ) of coinage metal dimers in the singlet ${ }^{1} \sum_{\mathrm{g}}^{+}$(panel a) and in the triplet ${ }^{3} \Sigma_{\mathrm{u}}^{+}$(panel b) states.

metal dimers, play an important role in the coinage metal highspin clusters.

Relativity is known to influence the atomic orbitals in two ways: A direct relativistic effect leads to contraction of the atomic s- and (to a lesser extent) p-orbitals accompanied by lowering of the orbital energy levels, and an indirect relativistic effect, which is due to the shielding of the nuclear charge by the inner core electrons, leads to expansion of the atomic d-orbitals accompanied by the respective destabilization of the orbital energy levels. ${ }^{39}$ A combination of both effects leads to a narrowing of the d-s energy gap in transition metals and to a widening of the $s-p$ gap in heavy p-elements. A textbook manifestation of the effect of relativity on the properties of chemical substances is the color of crystalline gold, for which the $5 d-6$ sp interband transition energy is reduced from $3.5 \mathrm{eV}$ in the nonrelativistic description to only $1.9 \mathrm{eV}$ when relativity is included. ${ }^{40}$

Direct and indirect relativistic effects are important for the stability of high-spin coinage metal dimers. Thus, a stabilization of the copper $4 \mathrm{~s}$-orbital and a destabilization of the copper $3 \mathrm{~d}$ orbitals should result in an increased participation of the atomic d-electrons in bonding as compared with the nonrelativistic description. Another important factor is the contraction of the valence s-orbital of copper atom due to relativity. This contraction leads to a noticeable shortening of the bond length in ${ }^{3} \mathrm{Cu}_{2}$ as obtained in the relativistically corrected all-electron calculations. Using the outer-valence Green's function (OVGF) formalism, ${ }^{41}$ the following one-electron orbital energies were obtained in the relativistic (nonrelativistic) calculations for atomic copper: $\epsilon_{3 \mathrm{~d}}^{\mathrm{R}}=-14.34 \mathrm{eV}\left(\epsilon_{3 \mathrm{~d}}^{\mathrm{NR}}=-14.38 \mathrm{eV}\right)$ and $\epsilon_{4 \mathrm{~s}}^{\mathrm{R}}=-7.64 \mathrm{eV}\left(\epsilon_{4 \mathrm{~s}}^{\mathrm{NR}}\right.$ $=-7.41 \mathrm{eV})$. Although the indirect relativistic effect on the copper $3 \mathrm{~d}$-orbitals is not strong, there is a noticeable 4 s-orbital stabilization which brings the relativistic value to a much better agreement with the experimentally measured ionization potential of $7.73 \mathrm{eV}$ of atomic copper. ${ }^{42}$ The results of the OVGF calculations carried out with the inclusion of relativity and at the nonrelativistic level of theory are presented in graphic form in Figure 2. In this figure, the narrowing down of the gap between the valence $\mathrm{s}$ and $\mathrm{d}$ orbitals upon the inclusion of relativity is clearly visible.
The results of the all-electron calculations of silver and gold dimers carried out at the relativistically corrected CCSD(T) level are reported in Table 3. In these calculations, the uncontracted basis sets of double- $\zeta$ and triple- $\zeta$ quality optimized by Dyall ${ }^{25}$ in the four-component relativistic atomic calculations were employed. However, for gold dimer, the use of the triple- $\zeta$ basis set was prohibitively costly in connection with the CCSD(T) formalism, thus only the calculations employing the double- $\zeta$ basis set were carried out. Extension of the basis set beyond triple- $\zeta$ quality was not feasible even for the silver dimers and consequently extrapolation to the complete basis set was not attempted. The results of $\operatorname{CCSD}(\mathrm{T})$ calculations with the triple- $\zeta$ basis set carried out with the account of BSSE and without such an account show a reasonable degree of convergence which suggests that the basis set incompleteness effects in these calculations are fairly weak. Because relativity plays an important role for elements as heavy as silver and gold and because the $\operatorname{CCSD}(\mathrm{T})$ calculations with the uncontracted basis sets are very time-consuming, the respective nonrelativistic calculations were not carried out.

The results of both sets, with account of BSSE and without account of BSSE, of the CCSD(T)/TZ calculations for silver dimer in the ${ }^{1} \sum_{\mathrm{g}}^{+}$state are in a reasonable agreement with the experimental data. This gives us confidence that the spectroscopic parameters of the triplet ${ }^{3} \Sigma_{\mathrm{u}}^{+}$state of $\mathrm{Ag}_{2}$ represent a fair estimate of the parameters of this molecule in the gas phase. The ${ }^{3} \mathrm{Ag}_{2} \mathrm{BDE}$ amounts to $0.74 \pm 0.03$ $\mathrm{kcal} / \mathrm{mol}$ which is in the same ballpark as the BDEs of the high-spin rubidium and cesium dimers, 0.633 and $0.706 \mathrm{kcal} /$ mol, respectively. ${ }^{10}$ This indicates that the role of valence d-electrons of silver is not very important in the ${ }^{3} \mathrm{Ag}_{2}$ dimer. Indeed, in silver atom, the effect of relativity which tends to stabilize valence s-orbitals and destabilize valence d-orbitals is largely compensated by the effect of shielding of the nuclear charge by the inner core electrons. ${ }^{39}$ Therefore, the gap between the valence $\mathrm{d}$ and s orbitals remains relatively wide, comparable to the copper atom. Comparison of the relativistic and nonrelativistic OVGF results for the valence one-electron energy levels of silver atom (see Figure 2) shows that the overall effect is relatively modest: the $5 \mathrm{~s}$ orbital is 
stabilized by less than $0.6 \mathrm{eV}$ from $\epsilon_{5 \mathrm{~s}}^{\mathrm{NR}}=-6.76 \mathrm{eV}$ to $\epsilon_{5 \mathrm{~s}}^{\mathrm{R}}=$ $-7.34 \mathrm{eV}$ and the $4 \mathrm{~d}$ orbital is destabilized by a comparable amount of energy from $\epsilon_{4 \mathrm{NR}}^{\mathrm{NR}}=-14.17 \mathrm{eV}$ to $\epsilon_{4 \mathrm{~d}}^{\mathrm{R}}=-13.61$ $\mathrm{eV}$. Because the covalent radius of silver atom is greater than that of copper, a longer $\mathrm{Ag}-\mathrm{Ag}$ bond length and consequently a weaker bond energy result.

Gold dimer is bonded considerably stronger than silver both in the singlet ${ }^{1} \Sigma_{\mathrm{g}}^{+}$ground-state and in the triplet ${ }^{3} \Sigma_{\mathrm{u}}^{+}$ excited state. Only the double- $\zeta$ quality basis set was affordable for gold dimers at the $\operatorname{CCSD}(\mathrm{T})$ level of theory, therefore the BSSE in the calculated spectroscopic parameters remains relatively high. The results obtained with the basis set of similar quality for the copper and silver dimers suggest that the BSSE corrected values provide a lower bound for BDE, whereas the uncorrected for BSSE values may deviate considerably from the converged value especially in the case of the triplet dimer.

Much stronger bonding in the triplet gold dimer can be explained by the greater role of the $5 \mathrm{~d}$ electrons in the nopair bond formation. Indeed, the effect of relativity in gold is fairly strong which is evidenced by a stabilization of the 6 s one-electron energy levels from $\epsilon_{6 \mathrm{~s}}^{\mathrm{NR}}=-6.74 \mathrm{eV}$ to $\epsilon_{6 \mathrm{~s}}^{\mathrm{R}}=$ $-8.88 \mathrm{eV}$ and by a destabilization of the $5 \mathrm{~d}$ energy levels from $\epsilon_{5 \mathrm{~d}}^{\mathrm{NR}}=-13.69 \mathrm{eV}$ to $\epsilon_{5 \mathrm{~d}}^{\mathrm{R}}=-11.87 \mathrm{eV}$ as obtained from the OVGF calculations (see Figure 2). Such a marked reduction in the width of the $d-$ s energy gap should result in a much stronger participation of the valence d-electrons in the bonding in ${ }^{3} \mathrm{Au}_{2}$. After the BSSE correction, the BDE of ${ }^{3} \mathrm{Au}_{2}$ amounts to $4.56 \mathrm{kcal} / \mathrm{mol}$ and may in reality be even greater than that value due to the incompleteness of the basis set employed in the calculations. This result suggests that the high-spin gold clusters may show an extremely strong no-pair bonding unprecedented in lighter elements.

\section{Conclusions}

Coinage metal dimers studied in the present article show fairly strong no-pair bonding in the high-spin states. The bond dissociation energy can reach as much as nearly $5 \mathrm{kcal} / \mathrm{mol}$ in ${ }^{3} \mathrm{Au}_{2}$ which is nearly an order of magnitude stronger than the dissociation energy of heavy alkali metal dimers ${ }^{3} \mathrm{Rb}_{2}$ and ${ }^{3} \mathrm{Cs}_{2} .{ }^{10}$ Such a strong bonding is really surprising provided that it can not be attributed to the spin-pairing mechanism as in usual chemical bond. ${ }^{4-7,16,8}$

From comparison with the alkali metal dimers, it is apparent that the valence d-electrons of coinage metal atom take part in formation of the no-pair bond in high-spin dimeric species. The bonding in ${ }^{3} \mathrm{Cu}_{2},{ }^{3} \mathrm{Ag}_{2}$, and ${ }^{3} \mathrm{Au}_{2}$ is therefore quite sensitive to an accurate account of the effect of relativity, because the latter alters considerably the oneelectron energies of atomic s and d electrons. The increase of the bonding energy in the triplet coinage metal dimers is clearly visible from Figure 3, where the variation of the bond dissociation energy in the singlet and triplet coinage metal dimers is plotted. The very strong no-pair bonding in the high-spin gold dimer is a clear manifestation of relativistic effect on the one-electron energies which reduces more than twice the $\mathrm{d}-\mathrm{s}$ energy gap (see also Figure 2).

Typically, the no-pair bonding in high-spin clusters is strongly nonadditive and the bond dissociation energy per atom grows rapidly with the cluster size..$^{4-7,16,8,10,11}$ In large clusters, the BDE/atom may reach values as high as $19 \mathrm{kcal} / \mathrm{mol}$ for highspin copper clusters. ${ }^{16}$ Considering that, in the dimeric form, ${ }^{3} \mathrm{Au}_{2}$ is bound twice as strong as ${ }^{3} \mathrm{Cu}_{2}$, this energy limit can be easily surpassed in bulky high-spin gold clusters. It might be expected that, in large gold clusters, the binding energy per atom can be well in excess of $20 \mathrm{kcal} / \mathrm{mol}$ which becomes comparable with the strength of usual chemical bonds.

\section{References and Notes}

(1) McAdon, M. H.; Goddard, W. A., III. J. Phys. Chem. 1988, 92, $1352-1365$ 466.

(2) Glukhovtsev, M. N.; Schleyer, P. v. R. Isr. J. Chem 1993, 33, 455-

(3) Hoffmann, R.; Shaik, S.; Hiberty, P. C. Acc. Chem. Res. 2003, 36, 750-756.

(4) Danovich, D.; Wu, W.; Shaik, S. J. Am. Chem. Soc. 1999, 121, $3165-3174$.

(5) de Visser, S. P.; Alpert, Y.; Danovich, D.; Shaik, S. J. Phys. Chem. A 2000, 104, 11223-11231.

(6) de Visser, S. P.; Danovich, D.; Wu, W.; Shaik, S. J. Phys. Chem. A 2002, 106, 4961-4969.

(7) de Visser, S. P.; Danovich, D.; Shaik, S. Phys. Chem. Chem. Phys. 2003, 5, 158-164.

(8) Alikhani, M. E.; Shaik, S. Theor. Chem. Acc. 2006, 116, 390-397.

(9) Lewis, G. N. J. Am. Chem. Soc. 1916, 38, 762-785.

(10) Soldán, P.; Cvitaš, M. T.; Hutson, J. M. Phys. Rev. A 2003, 67, 054702(1-4)

(11) Colavecchia, F. D.; Burke, J. P., Jr.; Stevens, W. J.; Salazar, M. R.; Parker, G. A.; Pack, R. T. J. Chem. Phys. 2003, 118, 5484-5495.

(12) Bradley, C. C.; Sackett, C. A.; Tollett, J. J.; Hulet, R. G. Phys. Rev. Lett. 1995, 75, 1687-1691.

(13) Anderson, M. H.; Ensher, J. R.; Matthews, M. R.; Wieman, C. E.; Cornell, E. A. Science 1995, 269, 198-201.

(14) Davis, K. B.; Mewes, M.-O.; Andrews, M. R.; van Druten, N. J.; Durfee, D. S.; Kurn, D. M.; Ketterle, W. Phys. Rev. Lett. 1995, 75, 39693973.

(15) Pereiro, M.; Baldomir, D.; Arias, J. E. Phys. Rev. A 2007, 75, 063204(1-6)

(16) de Visser, S. P.; Kumar, D.; Danovich, M.; Nevo, N.; Danovich, D.; Sharma, P. K.; Wu, W.; Shaik, S. J. Phys. Chem. A 2006, 110, 85108518.

(17) Higgins, J.; Hollebeek, T.; Reho, J.; Ho, T.-S.; Lehmann, K. K.; Rabitz, H.; Scoles, G.; Gutowski, M. J. Chem. Phys. 2000, 112, 57515761

(18) Brühl, F. R.; Miron, R. A.; Ernst, W. E. J. Chem. Phys. 2001, 115, 10275-10281.

(19) Schulz, C. P.; Claas, P.; Schumacher, D.; Stienkemeier, F. Phys. Rev. Lett. 2004, 92, 013401(1-4)

(20) Bondybey, V. E. J. Chem. Phys. 1982, 77, 3771-3772.

(21) Boys, S. F.; Bernardi, F. Mol. Phys. 1970, 19, 553-566.

(22) Balabanov, N. B.; Peterson, K. A. J. Chem. Phys. 2005, 123, 064107(1-15)

(23) (a) Feller, D. J. Chem. Phys. 1992, 96, 6104-6114; 1993, 98, 70597071. (b) Peterson, K. A.; Dunning, T. H., Jr. J. Chem. Phys. 1995, 102, 2032-2041.

(24) Helgaker, T.; Klopper, W.; Koch, H.; Noga, J. J. Chem. Phys. 1997, 106, 9639-9646.

(25) Dyall, K. G. Theor. Chem. Acc. 2007, 117, 483-489, Available from the Dirac web site, http://dirac.chem.sdu.dk.

(26) Dyall, K. G. J. Chem. Phys. 1997, 106, 9618-9626.

(27) Filatov, M.; Dyall, K. G. Theor. Chem. Acc. 2007, 117, 333-338.

(28) Kraka, E.; Gräfenstein, J.; Filatov, M.; Joo, H.; Izotov, D.; Gauss, J.; He, Y.; Wu, A.; Polo, V.; Olsson, L.; Konkoli, Z.; He, Z.; Cremer, D. COLOGNE08; Stocton CA: University of the Pacific, 2008.

(29) (a) Douglas, M.; Kroll, N. M. Ann. Phys. 1974, 82, 89-155. (b) Hess, B. A. Phys. Rev. A 1986, 33, 3742-3748.

(30) MOLPRO, version 2006. 1, a package of ab initio programs, , Werner, H.-J.; Knowles, P. J.; Lindh, R.; Manby, F. R.; Schutz, M.; Celani, P.; Korona, T.; Rauhut, G.; Amos, R. D.; Bernhardsson, A.; Berning, A.; Cooper, D. L.; Deegan, M. J. O.; Dobbyn, A. J.; Eckert, F.; Hampel, C.; Hetzer, G.; Lloyd, A. W.; McNicholas, S. J.; Meyer, W.; Mura, M. E., Nicklass, A.; Palmieri, P.; Pitzer, R.; Schumann, U.; Stoll, H.; Stone, A. J.; Tarroni, R.; Thorsteinsson, T. see http://www.molpro.net.

(31) Frisch, M. J.; Trucks, G. W.; Schlegel, H. B.; Scuseria, G. E.; Robb, M. A.; Cheeseman, J. R.; Montgomery, J. A., Jr.; Vreven, T.; Kudin, K. N., Burant, J. C.; Millam, J. M.; Iyengar, S. S.; Tomasi, J.; Barone, V.; Mennucci, B.; Cossi, M.; Scalmani, G.; Rega, N.; Petersson, G. A.; Nakatsuji, H.; Hada, M.; Ehara, M.; Toyota, K.; Fukuda, R.; Hasegawa, J.; Ishida, M.; Nakajima, T.; Honda, Y., Kitao, O.; Nakai, H.; Klene, M.; Li, X.; Knox, J. E.; Hratchian, H. P.; Cross, J. B.; Bakken, V.; Adamo, C.; Jaramillo, J.; Gomperts, R.; Stratmann, R. E.; Yazyev, O.; Austin, A. J.; Cammi, R.; Pomelli, C.; Ochterski, J. W.; Ayala, P. Y.; Morokuma, K.; Voth, G. A.; Salvador, P.; Dannenberg, J. J.; Zakrzewski, V. G.; Dapprich, S.; Daniels, A. D.; Strain, M. C.; Farkas, O.; Malick, D. K.; Rabuck, A. D.; Raghavachari, K.; Foresman, J. B.; Ortiz, J. V.; 
Cui, Q.; Baboul, A. G.; Clifford, S.; Cioslowski, J.; Stefanov, B. B.; Liu, G.; Liashenko, A.; Piskorz, P.; Komaromi, I.; Martin, R. L.; Fox, D. J.; Keith, T.; Al-Laham, M. A.; Peng, C. Y.; Nanayakkara, A.; Challacombe, M.; Gill, P. M. W.; Johnson, B.; Chen, W.; Wong, M. W.; Gonzalez, C.; Pople, J. A. Gaussian 03, revision C.02; Gaussian, Inc.: Wallingford, CT, 2004

(32) Straatsma, T. P.; Apr, E.; Windus, T. L.; Bylaska, E. J.; de Jong, W.; Hirata, S.; Valiev, M.; Hackler, M.; Pollack, L.; Harrison, R.; Dupuis, M.; Smith, D. M. A.; Nieplocha, J.; Tipparaju V.; Krishnan, M.; Auer, A. A.; Brown, E.; Cisneros, G.; Fann, G.; Frchtl, H.; Garza, J.; Hirao, K.; Kendall, R.; Nichols, J.; Tsemekhman, K.; Wolinski, K.; Anchell, J.; Bernholdt, D.; Borowski, P.; Clark, T.; Clerc, D.; Dachsel, H.; Deegan, M.; Dyall, K.; Elwood, D.; Glendening, E.; Gutowski, M.; Hess, A.; Jaffe, J.; Johnson, B.; Ju, J.; Kobayashi, R.; Kutteh, R.; Lin, Z.; Littlefield, R.; Long, X.; Meng, B.; Nakajima, T.; Niu, S.; Rosing, M.; Sandrone, G.; Stave, M.; Taylor, H.; Thomas, G.; van Lenthe, J.; Wong, A.; Zhang, Z. NWChem, A Computational Chemistry Package for Parallel Computers, version 5.0; Pacific Northwest National Laboratory: Richland, WA, 2007.

(33) Dolg, M.; Stoll, H.; Preuss, H.; Pitzer, R. M. J. Phys. Chem. 1993, 97, 5852-5859.

(34) Morse, M. D. Chem. Rev. 1986, 86, 1049-1109.
(35) (a) Simard, B.; Hacket, P. A.; Jemes, A. M.; Landridge-Smith, P. R. R. Chem. Phys. Lett. 1991, 186, 415-422. (b) Beutel, V.; Kramer, H. G.; Bhale, G. L.; Kuhn, M.; Weyers, K.; Demtroder, W. J. Chem. Phys. 1993, 98, 2699-2708.

(36) Huber, K. P.; Herzberg, G. Constants of Diatomic Molecules; Van Nostrand Reinhold: New York, 1979.

(37) Bominaar, E.; Guillin, J.; Marathe, V. R.; Sawaryn, A.; Trautwein, A. X. Hyperfine Interact. 1988, 40, 111-122.

(38) Monari, A.; Pitarch-Ruiz, J.; Bendazzoli, G. L.; Evangelisti, S.; Snachez-Marin, J. J. Chem. Theory Comput. 2008, 4, 404-413.

(39) Pyykkö, P. Chem. Rev 1988, 88, 563-594; 1997, 97, 597-636.

(40) Romaniello, P.; de Boeij, P. L. J. Chem. Phys. 2005, 122, 164303(1-6)

(41) (a) Cederbaum, L. S.; Domcke, W. Adv. Chem. Phys. 1977, 36, 205-344. (b) von Niessen, W.; Schirmer, J.; Cederbaum, L. S. Comput. Phys. Rep 1984, 1, 57-125.

(42) Ionization potentials of atoms and atomic ions. In CRC Handbook of Chemistry and Physics; Lide, D. R., Ed.; Taylor \& Francis Group: Boca Raton, FL, 1992; pp 10-211

JP803667N 